\title{
Scrub typhus in third trimester of pregnancy: dilemmas in diagnosis and management
}

\author{
Geeta Jain$^{1}$, Preeti Priyadarshani ${ }^{2 *}$, Garima Sisodia ${ }^{1}$
}

${ }^{1}$ Department of Obstetrics and Gynaecology, Government Medical College, Haldwani, UK
${ }^{2}$ Department of Obstetrics and Gynaecology, All India Institute of Medical Sciences, Gorakhpur, Uttar Pradesh

Received: 03 November 2020

Accepted: 09 December 2020

\section{*Correspondence:}

Dr. Preeti Priyadarshani,

E-mail: drpreeti.priyadarshani@gmail.com

Copyright: (c) the author(s), publisher and licensee Medip Academy. This is an open-access article distributed under the terms of the Creative Commons Attribution Non-Commercial License, which permits unrestricted non-commercial use, distribution, and reproduction in any medium, provided the original work is properly cited.

\begin{abstract}
Scrub typhus is an acute febrile illness rarely diagnosed in pregnancy. Here we report a case of scrub typhus in the third trimester of pregnancy, its associated complications and its management to ensure a favourable fetomaternal outcome. Mrs X, a 22- year old primigravida, was diagnosed with scrub typhus at 38 weeks of pregnancy after developing high- grade fever for 12 days. An atypical eschar was seen on one foot. Her ultrasonography revealed foetal tachycardia and severe oligohydramnios. She was started on tab azithromycin $500 \mathrm{mg}$ OD but remained febrile even after two days. Fever subsided at dose of $1 \mathrm{gm} /$ day. When she was afebrile for 48 hours and adequately hydrated, labour was induced and she delivered vaginally a full-term alive baby of $2.2 \mathrm{~kg}$. Vertical transmission was not observed in the baby. Scrub typhus is an easy-to-treat cause of acute undifferentiated febrile illness in the Indian subcontinent, and should always be a differential diagnosis even in the absence of a pathognomic eschar. Timely treatment ensures a favourable fetomaternal outcome.
\end{abstract}

Keywords: Scrub typhus, Third trimester, Oligohydramnios

\section{INTRODUCTION}

Acute febrile illness developing in pregnancy poses problems to the clinicians, both in diagnosing, and in management. Scrub typhus is a vector borne rural zoonosis endemic to the 'tsutsugamushi triangle', which includes the entire Indian subcontinent (Figure 1). ${ }^{1}$

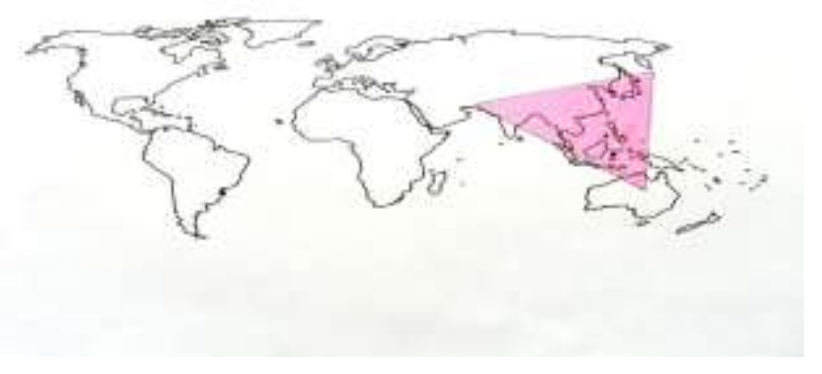

Figure 1: Tsutsugamushi triangle.
In spite of the endemic nature of disease and several epidemics reported from Indian states including Uttarakhand, many cases remain undiagnosed due to lack of suspicion. ${ }^{2}$ In fact, the authors could not find any published case of scrub typhus in pregnancy from the Kumaon region of Uttarakhand, where our tertiary medical college is situated.

\section{CASE REPORT}

Mrs X was 22-year-old primigravida receiving care at a private hospital and referred to us at 38 weeks' gestation with complaints of high-grade fever for past twelve days. It was associated with chills and rigors, body ache and dry cough. She did not have any history of skin rash, jaundice, joint pains, diarrhoea, urinary complaints or bleeding manifestations. On examination, she was conscious and oriented, but dyspnoeic and tachypnoeic. Her pulse rate was $110 \mathrm{bpm}$ and BP 114/56 mmHg. Her 
temperature was spiking up to 103-104-degree Fahrenheit, and only decreased with paracetamol administration. She had a small crusted papular lesion on one of her feet, which did not look like a typical eschar (Figure 2). She had not noticed the lesion before we pointed it to her.

Laboratory investigations were negative for dengue, malaria and typhoid. Haemogram showed haemoglobin $12 \mathrm{gm} \%$, PCV 36\%, leucocytosis of 16000 and platelet count of 1.28 lakh. Proteinuria of +1 was seen. Her ultrasonography showed a single live intrauterine pregnancy of 34 weeks with foetal tachycardia and severe oligohydramnios (AFI $1.2 \mathrm{~cm}$ ). All other examinations and investigations were within normal range. A provisional diagnosis of primigravida with full term pregnancy with oligohydramnios with pyrexia of unknown origin was made. Consent for poor maternofoetal prognosis was taken in view of high-grade temperature and severe oligohydramnios. Foetal monitoring was done with DFMC, NST twice a day, modified manning's test biweekly.

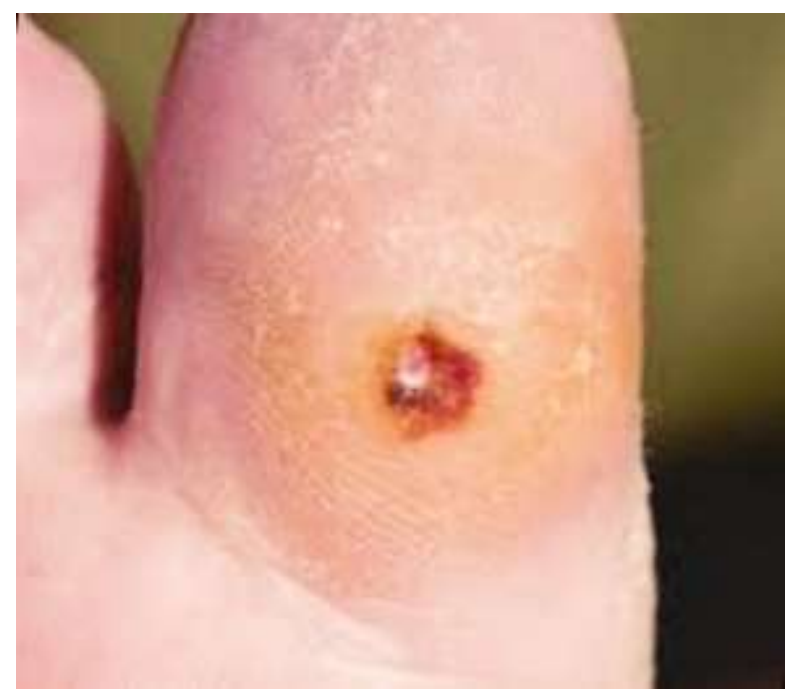

Figure 2: Atypical eschar.

She was empirically started on injection ceftriaxone $1 \mathrm{gm}$ I/V 12-hourly and injection paracetamol I/V 8-hourly. When she continued having high grade fever, her serum was tested for tsutsugamushi antibody (ICT) by Enzyme Immunoassay which was positive, after which she was started on tab azithromycin $500 \mathrm{mg}$ OD. She described symptomatic relief but continued to have high grade fever even after 48 hours. The dose of azithromycin was then increased to 1 gram/day after which the fever subsided. When she was afebrile for 48 hours and adequately hydrated, labour was induced with dinoprostone gel instilled intra-cervically. After 8 hours of labour with continued foetal monitoring, she delivered a full term alive male baby of $2.2 \mathrm{~kg}$. She was continued on tab. azithromycin 1 gram per day for 5 days after delivery, during which she remained afebrile. The infection was not vertically transmitted to the infant. Both the mother and baby were well at 2-weeks follow-up. Written informed consent was obtained from the patient for publication of her case report.

\section{DISCUSSION}

Scrub typhus is caused by Orientia tsutsugamushi, a small gram negative obligate intracellular organism, and is transmitted to humans by larvae (chiggers) of an arthropod vector of the Trombiculidae family. Humans are accidental hosts in this zoonosis. While nearly a million cases of scrub typhus are reported worldwide each year, it is rarely reported in pregnancy. ${ }^{3}$

The clinical features and the method of diagnosis of scrub typhus in pregnancy are similar to those of non-pregnant adults. ${ }^{3}$ The initial presentation is fever with headache and malaise, lymphadenopathy, rash and eschar. ${ }^{3,4}$ The infection causes disseminated vasculitis and perivascular inflammatory lesions resulting in vascular leak and end organ injury. ${ }^{5}$ All organs, including the lungs, liver, kidneys and brain are susceptible to damage even in the non-pregnant state. Laboratory studies usually reveal leucopenia, thrombocytopenia, deranged KFT and LFT, proteinuria and reticulonodular infiltrate on chest X-ray. In pregnancy, it is associated with increased foetal loss, preterm delivery and SGA infants. ${ }^{5}$ However, the exact impact of this infection on fetal outcome is still unclear. ${ }^{6,7}$ The mortality rate in untreated individuals can be as high as $30 \% .^{3,4}$ but we were able to achieve a good foetomaternal outcome in our patient.

Misdiagnosis and under diagnosis can occur due to lack of availability of diagnostic tests and the presence of nonspecific symptoms, especially in the absence of a characteristic eschar. The eschar forms at the site of tick bite and is pathognomic of scrub typhus, but is uncommon in South East Asian patients. ${ }^{5}$ It may also be missed in dark skinned individuals. ${ }^{4,5}$ Diagnosis and decision to initiate treatment should be based on clinical suspicion and confirmed by serological testing.

The recommended standard treatment of scrub typhus in non-pregnant adults is doxycycline. ${ }^{8}$ However, it is classified as a category D drug, and is contraindicated in pregnancy due to the associated foetal risks. Chloramphenicol, a category $\mathrm{C}$ drug, may be given in late trimesters of pregnancy with caution. ${ }^{5}$ Azithromycin, a macrolide antibiotic, is a category B drug and has emerged as the drug of choice during pregnancy and lactation. ${ }^{3,8}$ Various doses of azithromycin have been recommended, the most common being $500 \mathrm{mg}$ OD for 3-5 days. ${ }^{3}$ Some studies have reported successful treatment with $500 \mathrm{mg}$ single dose..$^{3,5-7,9}$ However, our patient required a higher dose of 1 gram per day for a longer duration for her fever to subside. It could be because the diagnosis was delayed in our case by almost two weeks from the onset of symptoms. Several other authors have reported the need for such high doses in pregnant women diagnosed with scrub typhus. ${ }^{9}$ It is 
preferable to continue treatment for at least three days after fever subsides and until there is evidence of clinical improvement to avoid relapse. ${ }^{10}$ Rigorous supportive management prevents progression to DIC and septic shock.

Our patient was in the third trimester of pregnancy when she was diagnosed with scrub typhus. The patient in our case developed severe oligohydramnois with fetal tachycardia, suggestive of distress to the foetus in utero. It could be due to vasculopathy affecting the placental vessels and consequent circulatory impediment, but the exact mechanism is not clear. ${ }^{5,11}$ The patient was delivered vaginally after explaining high fetomaternal risks associated with caesarean and vaginal delivery and extensive discussions with the patient and her family, in view of her poor general condition. The baby was observed to rule out vertical transmission before being discharged to the mother. Neonatal scrub typhus has previously been reported in two cases, but the route of transmission was unclear in them. ${ }^{12,13}$ More reporting of diagnosed cases in pregnancy is required to gather extensive data on the effects of the disease on the foetus in utero.

\section{CONCLUSION}

Scrub typhus is an easy-to-treat cause of acute undifferentiated febrile illness in the Indian subcontinent, and should always be a differential diagnosis even in the absence of a pathognomic eschar. A high index of suspicion is required for its prompt diagnosis and management. Early diagnosis and treatment results in a favourable maternal and foetal outcome.

Funding: No funding sources

Conflict of interest: None declared

Ethical approval: Not required

\section{REFERENCES}

1. Rajan SJ, Sathyendra S, Mathuram AJ. Scrub typhus in pregnancy:Maternal and fetal outcomes. Obstetric Medicine. 2016;9(4):164-6

2. Peesapati N, Lakkapragada R, Sunitha S, Sivaram P V. Clinical manifestations and complications of scrub typhus: A hospital-based study from North Andhra. Astrocyte. 2015;2:116-20.
3. Kumar R, Thakur S, Bhawani R, Kanga A, Ranjan A. Clinical Profile of Scrub Typhus in Pregnancy in Sub-Himalayan Region. J Obstet Gynecol Ind. 2016; 66(S1):S82-7

4. WHO Recommended Surveillance Standards. Second edition. Available at: https://www.who.int/csr/resources/publications/surve illance/whocdscsrisr992.pdf. Accessed on 18 February 2020.

5. Vaddadi S, Remella VV, Vaddadi RS. Scrub typhus during pregnancy. J Clin Sci Res. 2016;5:124-6.

6. Kim YS, Yun HJ, Shim SK, Koo SH, Kim SY, Kim S. A comparative trial of a single dose of azithromycin versus doxycycline for the treatment of mild scrub typhus. Clin Infect Dis. 2004; 39(9):132935.

7. Kim YS, Lee HJ, Chang M, Son SK, Rhee YE, Shim SK. Scrub typhus during pregnancy and its treatment: a case series and review of the literature. Am J Trop Med Hyg. 2006;75(5):955-9

8. Lee SC, Cheng YJ, Lin CH, Lei WT, Chang HY, Lee $\mathrm{MD}$, et al. Comparative effectiveness of azithromycin for treating scrub typhus: A PRISMAcompliant systematic review and meta-analysis. Medicine. 2017;96(36).

9. Kim ES, Chung MH, Kang JS. Treatment of scrub typhus during pregnancy: review of Korean patients. Infect Chemother. 2010;40:130-1.

10. Information for Health Care Providers- Scrub typhus. Page last reviewed: August 2, 2020 From: https://www.cdc.gov/typhus/healthcareproviders/inde x.html. Accessed on 10 February 2020.

11. Paris DH, Chansamouth V, Nawtaisong $P$, Lowenberg EC, Phetsouvanh R. Coagulation and inflammation in scrub typhus and murine typhus-a prospective comparative study from Laos. Clin Microbiol Infect. 2012;18:1221-8.

12. Wang CL, Yang KD, Cheng SN, Chu ML. Neonatal scrub typhus: a case report. Pediatrics. 1992;89(5):965-8.

13. Suntharasaj T, Janjindamai W, Krisanapan $\mathrm{S}$. Pregnancy with scrub typhus and vertical transmission: a case report. J Obstet Gynaecol Res. 1997;23:75-8.

Cite this article as: Jain G, Priyadarshani P, Sisodia G. Scrub typhus in third trimester of pregnancy: dilemmas in diagnosis and management. Int J Reprod Contracept Obstet Gynecol 2021;10:413-5. 